\title{
On Some Instability Properties of Multi-Level Geostrophic Models
}

\author{
By S. Matsumoto \\ Meteorological Research Institute \\ (Manuscript received 5 February 1962)
}

\begin{abstract}
Instability properties are compared among multi-level geostrophic models with various number of freedom, in order to obtain some informations concerning the number of levels by which the baroclinic development is well forecast within the hierarchy of quasi-geostrophic models. The growth rate is primarily a function of static stability applied and is not affected very much by the interaction between layers. The effect of horizontal gradient of static stability is also studied numerically and the convex type distribution of the vertical profile of zonal flow is shown to have a destabilizing effect on certain type of disturbances.
\end{abstract}

A multiple parametric resolution is compared with corresponding multiple level resolution.

\section{Introduction}

There have been two different approaches in improving numerical weather prediction models. One is the introduction of nongeostrophic effects by means of balance equation or primitive equation which are considered as a higher approximation of atmospheric models (e.g., Charney, 1962). On the other hand, there still remain basic problems within the hierarchy of the quasi-geostrophic model and some improvements have been achieved especially in the forecast of baroclinic development by the use of multi-level quasi-geostrophic model (e.g., Saito, 1962).

The geostrophic adjustment is undoubtedly one of the most important procedures operating in the atmosphere especially for larger scale disturbances. In the mean time, the baroclinic development of cyclone scale having a great influence on the weather seems to be predicted at least qualitatively even by a simple baroclinic quasi-geostrophic model. There are still many problems unsolved concerning the subject what can be predicted by the quasi-geostrophic models as the first approximation of the atmosphere.

As a numerical prediction technique, we are facing with a problem how to describe the baroclinic structure within the limited number of freedom. There are two kinds of representation, i.e., multiple level model and multiple parameter model. As far as the number of freedom is small, it can be easily shown that any of those baroclinic models have almost the same character at least concerning their instability property (e.g., Eliassen, 1953). However, when the higher order effects are to be introduced, more detailed knowledge would be needed concerning the subjects such as how to choose information levels or what kind of functions would provide the best fit to the baroclinic structure within a limited number of freedom.

\section{The growth rate and the number of verti- cal resolution}

Let us divide the atmosphere into $K$ layers with a pressure depth of $\Delta p$ and denote the quantities on the middle of the $k$ th layer and those on the $k$ th intersections by subscripts $k$ 's and $k+1 / 2$ 's as is shown in Fig. 1 . To simplify the matter, we will here consider a perturbation in one direction $(x)$ superposing on a general flow $U_{k}$ and neglect the variation in $y$ direction except that so-called $\beta$ effect and the temperature gradient in $y$ direction

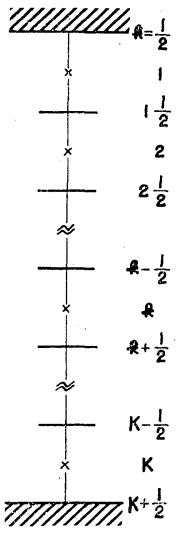

Fig. 1 
which generates the zonal flow are taken into consideration. After linearization the quasi-geostrophic model is specified by the equations

$$
\begin{gathered}
\frac{\partial^{3} z_{k}}{\partial t \partial x^{2}}+U_{k} \frac{\partial^{3} z_{k}}{\partial x^{3}}+\beta \frac{\partial z_{k}}{\partial x}-\left(w_{k+1 / 2}-w_{k-1 / 2}\right)=0 \\
(k=1,2, \cdots, K) \\
\begin{array}{c}
\frac{\partial\left(z_{k+1}-z_{k}\right)}{\partial t}+U_{k+1 / 2} \frac{\partial\left(z_{k+1}-z_{k}\right)}{\partial x} \\
-\left(U_{k+1}-U_{k}\right) \frac{\partial z_{k+1 / 2}}{\partial x}+\sigma_{k+1 / 2} w_{k+1 / 2}=0 \\
(k=1,2, \cdots, K-1)
\end{array}
\end{gathered}
$$

where

$$
\begin{aligned}
& w_{k+1 / 2}=\frac{f^{2}}{g \Delta p} \omega_{k+1 / 2} \\
& \sigma_{k+1 / 2}=\frac{g}{f^{2}} \frac{\partial z}{\partial p} \frac{\partial \ln \theta}{\partial p}(\Delta p)^{2}
\end{aligned}
$$

$z$ is the pressure height, $\omega$ the vertical $p$ velocity, $f$ the Coriolis parameter, $g$ the acceleration of gravity and $\theta$ the potential temperature. Eliminating the time dependent term from eqs. (1) and (2), we have so-called $\omega$-equation,

$$
\begin{gathered}
w_{k+1 \frac{1}{2}}-2 w_{k+1 / 2}+w_{k-1 / 2}+\sigma_{k+1 / 2} \frac{\partial^{2}}{\partial x^{2}} w_{k+1 / 2} \\
=\left(U_{k+1}-U_{k}\right) \frac{\partial^{3}}{\partial x^{3}}\left(z_{k+1}+z_{k}\right) \\
+\frac{1}{2}\left(U_{k+1}-2 U_{k+1 / 2}+U_{k}\right) \cdot \frac{\partial^{3}}{\partial x^{3}}\left(z_{k+1}\right. \\
\left.-z_{k}\right)+\beta \frac{\partial}{\partial x}\left(z_{k+1}-z_{k}\right) \\
\quad(k=1,2, \cdots, K-1)
\end{gathered}
$$

The system of equations the vorticity equation (1) and the adiabatic equation (2), is equivalent to the system of the vorticity equation (1) and the $\omega$-equation (3), and the latter system is prefered in the following discussions as in most of numerical weather prediction models.

Among characteristic features of baroclinic models, the growth rate of disturbances have been studied by many authors as a verification of representativeness of their models concerning the baroclinic behavior. Here let us, first of all, consider an atmosphere with uniform static stability to see to what extent the growth rate is modified simply by changing the number of layers $K$, because we can easily obtain the analitical solutions.

Assuming a simple harmonic wave distur- bance with a wave length $L$, the vertical velocity $w_{k+1 / 2}$ 's are expressed in terms of linear combination of $z_{k}$ 's as a solution of simultaneous equations (3). Eq. (1) can then be regarded as simultaneous equations of first order ordinary differential equations with respect to time having $K$ independent variables $z_{k}$ 's. Ogura (1957) showed the structure of disturbances by solving the simultaneous equations as an initial value problem in case of two level representation. Generally we have thereupon $K$ characteristic values corresponding to the number of freedom, which determine the mode of variation. The determinant generating the characteristic values is, however, essentially the same as the so-called frequency equation which is obtained by assuming a harmonic wave type solution, $i . e$. , $z_{k}, w_{k+\frac{1}{2}} \propto \exp [i l(x-c t)]$ where $l=2 \pi / L$.

Now let us further simplify our model by neglecting $\beta$ effect and higher deviations in the zonal wind profile. Thus eqs. (1) and (3) are rewritten into

$$
\begin{array}{r}
i l^{3}\left(c-U_{k}\right) z_{k}=w_{k+1 / 2}-w_{k-1 / 2} \\
(k=1,2, \cdots, K) \quad(4) \\
w_{k+1 \frac{1}{2}}-(2+\kappa) w_{k+1 / 2}+w_{k-1 / 2}=i l^{2} \cdot 2 \Delta\left[z_{k+1}+z_{k}\right] \\
(k=1,2, \cdots, K-1) \quad(5)
\end{array}
$$

where $\kappa=\sigma_{k+\frac{1}{2}} l^{2}$ gives constant static stability and $\Delta=U_{k}-U_{k+1 / 2}$ gives constant vertical wind shear. The boundary condition is as in the general cases

$$
w_{1 / 2}=w_{k+1 / 2}=0
$$

We can construct the frequency equation also in the following way. Applying (1) successively, we obtain with the aid of the upper boundary condition $w_{1 / 2}=0$,

$$
\begin{aligned}
w_{k+1 / 2} & =i l^{3}\left(c-U_{k}\right) z_{k}+w_{k-1 / 2} \\
& =i l^{3}\left[\left(c-U_{k}\right) z_{k}+\left(c-U_{k-1}\right) z_{k-1}+\cdots\right] \\
& =\sum_{k=1}^{k}\left(c-U_{k}\right) z_{k} \quad(k=1,2, \cdots, K-1)
\end{aligned}
$$

Substitution of (7) into (5) then gives

$$
\begin{aligned}
& \left(c-U_{k+1}-2 \Delta\right) z_{k+1}-\left(c-U_{k}+2 \Delta\right) z_{k} \\
& \quad-\kappa \sum_{k=1}^{k}\left(c-U_{k}\right) z_{k}=0 \quad(k=1,2, \cdots, K-1)
\end{aligned}
$$

together with

$$
\sum_{k=1}^{K}\left(c-U_{k}\right) z_{k}=0
$$


which specifies the lower boundary condition. We now have $K$ equations of independent variables $z_{k}$ 's and the condition of getting significant solution is that the determinant of the coefficients in eqs. (8) and (9) vanishes.

The frequency equations for our simple model with smaller numbers of freedom are

$$
\begin{aligned}
& (c-U)^{2}+\frac{2-\kappa}{2+\kappa} \Delta^{2}=0 \\
& (c-U)^{3}+\frac{2-2 \kappa-\kappa^{2}}{3+4 \kappa+\kappa^{2}} \cdot 4 \Delta^{2}(c-U)=0 \\
& (c-U)^{4}-\left[\frac{5}{4}+\frac{2+4(2+\kappa)-3(2+\kappa)^{2}-2(2+\kappa)^{3}}{2(2+\kappa)\left(2+4 \kappa+\kappa^{2}\right)^{2}}\right] \\
& \cdot 8 \Delta^{2}(c-U)^{2}+\left[\frac{9}{16}+\frac{2-2(2+\kappa)-3(2+\kappa)^{2}}{2(2+\kappa)\left(2+4 \kappa+\kappa^{2}\right)}\right] \\
& .16 \Delta^{4}=0
\end{aligned}
$$

where the first equation is for the 2-level model, the second for the 3-level model and the third for the 4-level model. As is well known, the real part of $c$ represents the phase velocity and the imaginary part corresponds to the growth rate of disturbances. Eq. (10. 1) is quite familiar and shows a characteristic feature of baroclinic instability; i.e., an amplifying mode does exist in case $\kappa<2$. In other words, longer waves are to be amplified in a stable stratification if the wave length is longer than a certain critical value which is a function of static stability having smaller value for less stable atmosphere. In an unstable stratification on the other hand, there seems to be a possibility to have stable disturbances in very short wave regime granted that the quasi-geostrophic balance is still guaranteed.

The growth rates of 2-level, 3-level and 4-level model are compared in Fig. 2 as a

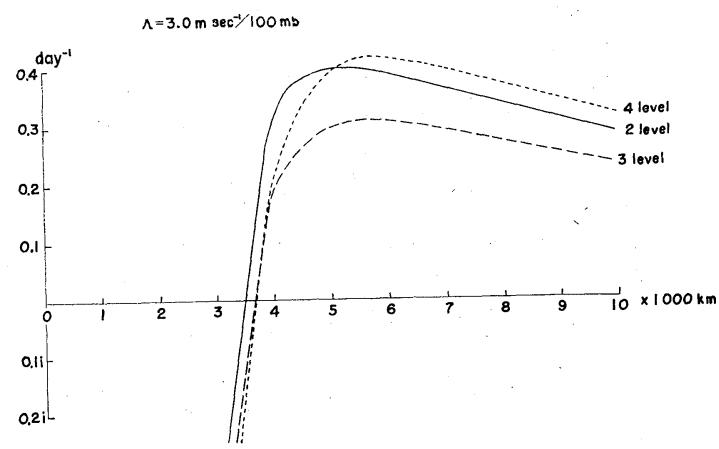

Fig. 2 The growth rate as a function of wave length for each of 2-level, 3-level and 4-level quasi-geostrophic model (unit in day). function of wave length which, of course, changes with static stability. The wind shear and the static stability are more or less arbitrary and here the wind shear of $3 \mathrm{~m}$ $\mathrm{sec}^{-1} / 100 \mathrm{mb}$ and the static stability at $500 \mathrm{mb}$ level of the standard atmosphere are chosen as a realistic value. The growth rate $\nu=c_{i} l$ $\left(c_{i}\right.$ is the imaginary part of $c$ ) is expressed in a unit of day. Stable disturbances which give real values of $c$ are also shown on Fig. 2 conveniently by giving negative value for $\nu$ with the same unit. It is seen that the growth rate changes with the number of resolution. Changes are also noticed on the critical wave length which divides unstable waves from stable waves and on the most unstable wave length having maximum growth rate. However, those changes do not seem to be so significant that we can expect essential modification simply by increasing the number of resolution.

It should be pointed out that only the most unstable mode is presented on Fig. 2 for each of the models. There are as many number of modes as the number of freedom. Therefore if we apply the larger number of levels, the baroclinic development that would be observed in the course of actual numerical integrations can be of a more manifold character, corresponding to the complexity of initial conditions.

\section{Baroclinic instability of multi-level model and the static stability distribution}

The static stability is not uniformly distributed in the actual atmosphere, but changes to a large extent from layer to layer. It is known that the vertical distribution of static stability $\sigma$ is inversely proportional to the square of pressure approximately. As is mentioned briefly in the previous section, the critical wave length, the maximum growth rate and the wave length associated with it are all related directly to the value of static stability.

It is quite a troublesome job to find out analytical solutions of models with larger number of levels, especially when the static stability is not uniformly distributed. Therefore, in this section, the matter will be dealt with numerically so as to see the effect of vertical distribution of static stability. The 
fundamental equations will be again (1) and (3), in which $\beta$ effect and the second derivative in the vertical distribution of $U_{k}$ are taken into account.

Let us consider a single harmonic wave with the wave length $L$. Then we have

$$
\left.\begin{array}{c}
z_{k}=z_{k}^{1} \cos \frac{2 \pi x}{L}+z_{k}^{2} \sin \frac{2 \pi x}{L} \\
w_{k+1 / 2}=w_{k+1 / 2} \cos ^{2 \pi x} \frac{2 \pi x}{L}+w_{k+1 / 2}^{2} \sin \frac{2 \pi x}{L}
\end{array}\right\}
$$

where superscript 1 and 2 show the amplitudes of cosine and sine component respectively. Substituting (11) to (1) and putting cosine term and sine term zero separately, we obtain the prognostic equation

$$
\begin{aligned}
\frac{\partial}{\partial t} z_{k}^{1,2}=\mp\left(\frac{2 \pi}{L} U_{k}-\frac{L}{2 \pi} \beta\right) z_{k}^{2,1} & \\
- & \frac{L^{2}}{4 \pi^{2}}\left(w_{k+1 / 2}^{1,2}-w_{k-1 / 2}{ }^{1,2}\right) \\
& (k=1,2, \cdots, K) \quad(12)
\end{aligned}
$$

and similarly we obtain the diagnostic equation from (3), i.e.

$$
\begin{aligned}
w_{k+1 \frac{1}{2}}^{1,2} & -\left(2+\kappa_{k+1 / 2}\right) w_{k+1 / 2}^{1,2}+w_{k-1 / 2}^{1,2} \\
& = \pm \frac{8 \pi^{3}}{L^{3}}\left[\left(U_{k+1}-U_{k}\right)\left(z_{k+1}^{2,1}+z_{k}^{2,1}\right)\right. \\
& \left.+\frac{1}{2}\left(U_{k+1}-2 U_{k+1 / 2}+U_{k}\right)\left(z_{k+1}^{2,1}-z_{k}^{2,1}\right)\right] \\
& \pm \frac{2 \pi}{L} \beta\left(z_{k+1}{ }^{2,1}-z_{k}^{2,1}\right),(k=1,2, \cdots, K-1)
\end{aligned}
$$

together with the boundary condition

$$
w_{1 / 2}^{1,2}=w_{K+1 / 2^{1,2}}=0
$$

where $\kappa_{k+1 / 2}=\sigma_{k+1 / 2} 4 \pi^{2} L^{-2}$ and double superscript and double sign are used in such a way that either first superscript or second is to be taken corresponding to upper sign or lower sign respectively.

The vertical distribution of static stability and general flow is assumed as follows :

$$
\begin{aligned}
\kappa_{k+1 / 2} & =\kappa_{K+1 / 2}\left(\frac{p_{K+1 / 2}}{p_{k+1 / 2}}\right)^{2} \\
U_{k} & =U_{m}+\Lambda\left(p_{m}-p_{k}\right)+\Lambda^{\prime}\left(p_{m}-p_{k}\right)^{2}
\end{aligned}
$$

where $\kappa_{K+1 / 2}=\left(\frac{R}{c_{p}}\right)^{2} \frac{4 \pi^{2}}{K^{2}} \frac{g}{f^{2} L^{2}} \frac{T}{\Gamma} \frac{\Gamma-\gamma}{\Gamma}(T$ is the surface temperature, $\Gamma$ the adiabatic lapse rate and $\gamma$ the lapse rate at the lowest layer) measures the static stability at the surface, $U_{m}$ is the general flow at $500 \mathrm{mb}\left(p_{m}=500 \mathrm{mb}\right)$, $\Lambda$ the vertical wind shear and $\Lambda^{\prime}$ measurs the curvature in the vertical profile of the general flow (positive for the concave type distribution). Because the general flow is in geostrophic balance, $\Lambda$ and $\Lambda^{\prime}$ are obviously associated with the temperature gradient and the static stability gradient normal to the direction of general flow.

Integrations were performed in a similar procedure as the ordinary numerical weather forecasting technique for various $L$ and $A$ assuming the same static stability given by $(14)^{*}$. Prognostic $\omega$-equation (13) is regarded as simultaneous equations with $K$ independent variables and can be solved either by use of reciprocal matrix or by successive substitution method given in appendix A. Because our interest is concentrated to a comparison of instability diagram, the initial condition of the integration may be chosen somewhat

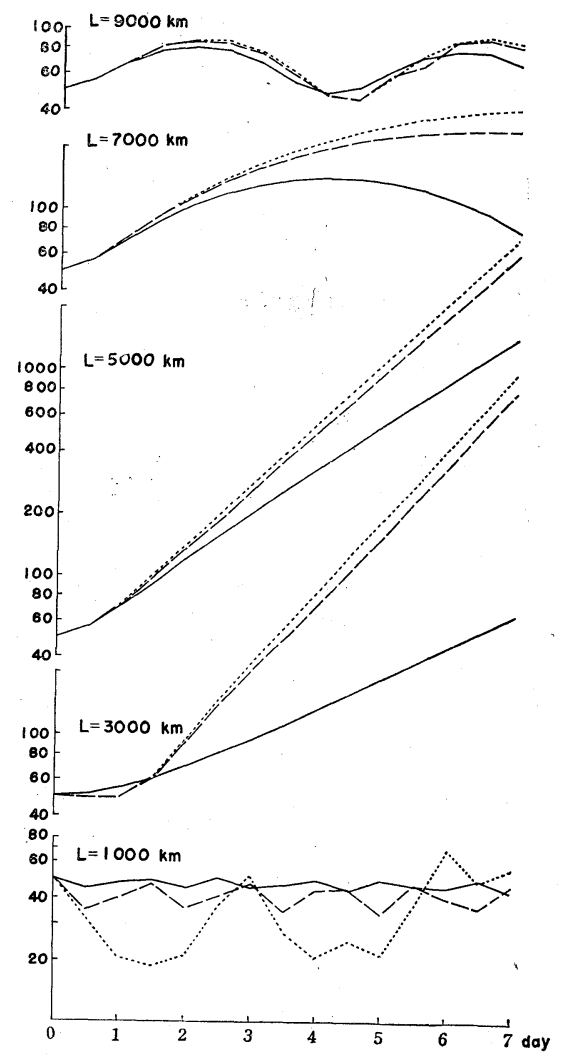

Fig. 3 The time change of kinetic energy of disturbance in a baroclinic atmosphere having the vertical wind shear of $4.0 \mathrm{~m} \mathrm{sec}^{-1} / 100 \mathrm{mb}$. The ordinate is the amplitude in a logarithmic scale and the abscissa is time. The amplitude of disturbance on $400 \mathrm{mb}$ is shown for each of 2 -level model (full line), 4-level model (broken line) and 8-level model (dotted line).

with $\gamma=0.6^{\circ} \mathrm{C} / 100 \mathrm{~m}$ 
arbitrarily and a disturbance having the same amplitude and the same phase angle is given initially on each of the levels.

Fig. 3 shows how the eddy kinetic energy change differs when the number of levels is increased for our model. The amplitude of disturbance on $400 \mathrm{mb}$ level is shown as a function of time for each of 2-level, 4-level and 8-level model. Examples shown in Fig. 3 compare the disturbances with the wave length of $1000 \mathrm{~km}, 3000 \mathrm{~km}, 5000 \mathrm{~km}, 7000 \mathrm{~km}$ and $9000 \mathrm{~km}$ superposing on a general flow having the vertical shear of $4.0 \mathrm{~m} \mathrm{sec}^{-1} / 100 \mathrm{mb}$. The exponential growth of unstable wave appear on this figure as a straight line and the

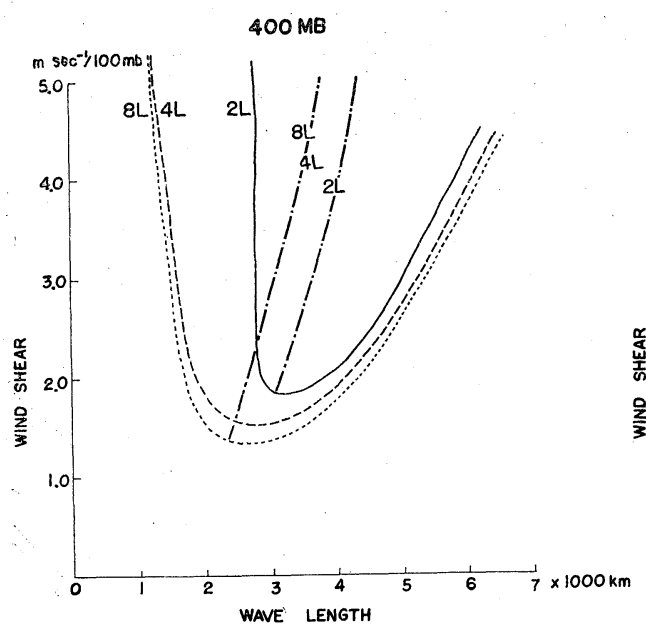

Fig. 4 The instability diagram of disturbance on $400 \mathrm{mb}$ level (the left figure) and on $800 \mathrm{mb}$ level (the right figure). The full lines are for 2-level model, the broken lines for 4-level model and the dotted lines for 8-level model. The chain lines indicate the most unstable disturbances.

waves are always stable because of the stabilizing effects of stable stratification and so-called $\beta$ effect respectively.

It is seen from Fig. 4 that the instability regime is expanded with increasing the number of levels. In other words, the disturbance of $L=2000 \mathrm{~km}$, for example, which does not develop with the use of 2-level model does develop with the use of 4 - or 8-level model, and further smaller disturbance of $L=1000 \mathrm{~km}$ develops in the lower atmosphere when 8-level model is applied. The most unstable wave length, on the other hand, becomes the smaller, the larger number of levels is introduced to our model. Those circumstances are undoubtedly related to the stables wave is discriminated by a periodic oscillation. The growth rate is thus easily evaluated from such diagrams.

Fig. 4 summarizes the results of experimental integrations in the form of instability diagram. Three curves in Fig. 4 indicate instability regimes of 2-level model, 4-level model and 8-level model in which the maximum instability is given by broken line. The left figure is estimated for disturbances on $400 \mathrm{mb}$ level and the right figure for disturbances on $800 \mathrm{mb}$ level. As is well known the instability does not take place unless the vertical wind shear exceeds a certain value and shorter waves and extremely longer

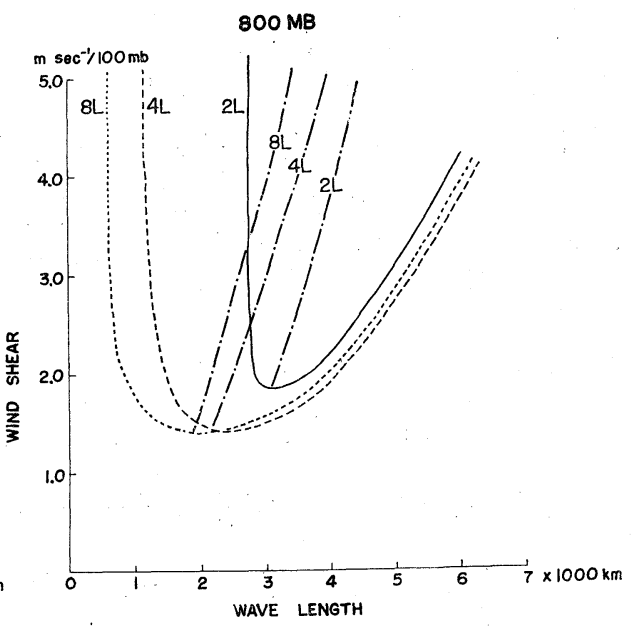

fact that the effect of low static stability in the lower atmosphere is taken into consideration when the atmosphere is divided into larger number of layers.

A comparison between the upper level instability diagram (the left figure) and the lower level instability diagram (the right figure) in Fig. 4 gives some informations concerning the interaction between layers. Of course 2-level model has only one static stability the value of which is that on $500 \mathrm{mb}$ and, therefore, the instability diagram is exactly the same between upper and lower level. As to 4-level model having three static stability values, the instability diagram changes but slightly, showing the influence 
of relatively low static stability in the lower layer. 8-level model having much better representation of the vertical stratification shows soemwhat different character in the instability diagram between upper and lower level, which means that the extremely less stable stratification in the lowermost part of the atmosphere has almost nothing to do with disturbances in upper atmosphere. It is concluded from the experimental knowledge on the most unstable wave that the scale of predominating disturbance is smaller in the lower atmosphere while long wave disturbances predominate in the higher atmosphere. Although the suggestion given above is derived basing on the linearized theory, it is verified by a numerical experiment with the use of 5-level quasi-geostrophic model (Matsumoto, 1962).

Some remarks should be mentioned briefly concerning the behavior of disturbance in stable regime. It is obvious from the conception of frequency equation that there are as many number of phase velocities as the number of freedom. Thus, in a baroclinic atmosphere, the disturbance on each level may generally have different phase velocity, which in turn generates energy transformation between eddy kinetic energy and potential energy. In this way, the amplitude of disturbance can change even in the stable regime.

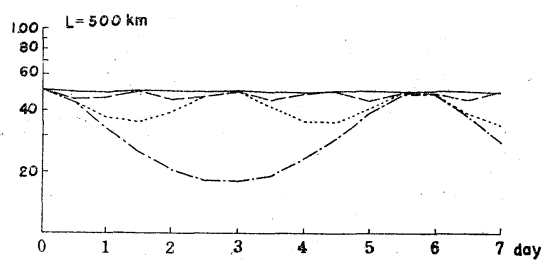

Fig. 5 The time change of amplitude of disturbance in stable regime. The wave length is $500 \mathrm{~km}$ and the vertical wind shear is $2.0 \mathrm{~m} \mathrm{sec}^{-1} / 100 \mathrm{mb}$. The full line is for 2-level model, the broken line for 4-level model, the dotted line for 8-level model ann the chain line for 16 -level model.

Fig. 5 shows how the amplitude of disturbance at $400 \mathrm{mb}$ with a wave length of $500 \mathrm{~km}$ actually changes periodically with time in a baroclinic atmosphere with the vertical wind shear of $2.0 \mathrm{~m} \mathrm{sec}^{-1} / 100 \mathrm{mb}$. It is clearly seen that the period of amplitude change is almost doubled when the number of vertical resolution is doubled. Such a remarkable difference would be originated from the confinement of models, i.e., how many vertical modes are allowed to describe the structure of disturbance, and the period itself would probably be related to the time in which all of the modes considered will come back to the initial situation again. In the actual atmosphere, the number of vertical modes might be limited within a certain number corresponding to the horizontal scale of disturbance because of dissipating effects. It is not yet cleared wether or not this fine structure has any important roles on the acuracy of numerical weather prediction.

Finally, let us discuss the effect of nonuniform vertical wind shear by making some additional integrations with $\Lambda^{\prime}$ included. Fig. 6 shows amplitude changes of disturbance with the wave length of $1000 \mathrm{~km}$ embedded in a baroclinic flow with $\Lambda=4.0 \mathrm{~m} \mathrm{sec}-1 / 100 \mathrm{mb}$ and $\Lambda^{\prime}=-0.25 \mathrm{~m} \mathrm{sec}^{-1} /(100 \mathrm{mb})^{2}$. Comparing Fig. 6 with the figure given in the lowermost

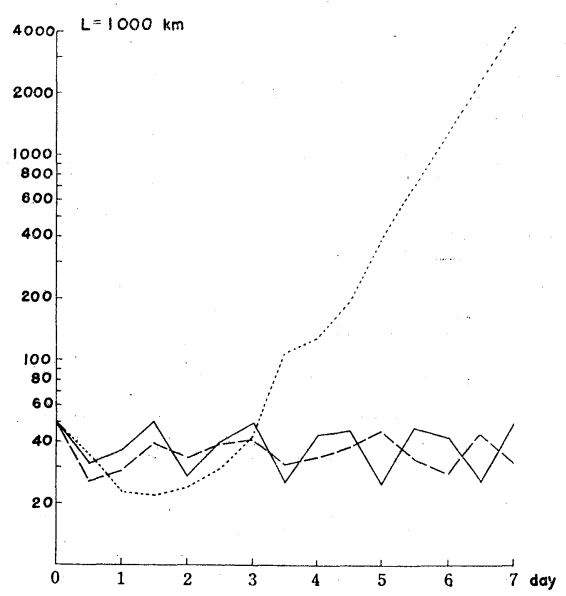

Fig. 6 The amplitude change of disturbance with the wave length of $1000 \mathrm{~km}$ embedded in a baroclinic flow with $\Lambda=4.0 \mathrm{~m} \mathrm{sec}^{-1} / 100$ $\mathrm{mb}$ and $\Lambda^{\prime}=-0.25 \mathrm{~m} \mathrm{sec}-1 /(100 \mathrm{mb})^{2}$. The full line is for 2-level model, the broken line for 4-level model and the dotted line for 8level model.

part of Fig. 3, it is clear that negative value of $\Lambda^{\prime}$ has a destabilizing effect. On the other hand, the effect of positive value of $\Lambda^{\prime}$ is shown in Fig. 7 for the case with $L=2000 \mathrm{~km}$, $\Lambda=3.0 \mathrm{~m} \mathrm{sec}^{-1} / 100 \mathrm{mb}$ and $\Lambda^{\prime}=0.25 \mathrm{~m} \mathrm{sec}^{-1} /(100$ $\mathrm{mb})^{2}$. Here again the amplitude change is given for a disturbance at $400 \mathrm{mb}$ level, which is found to be in stable regime. As is seen 


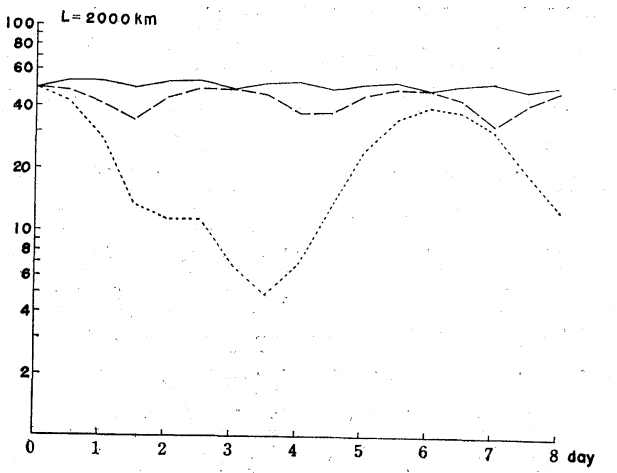

Fig. 7 The same as Fig. 6 for $L=2000 \mathrm{~km}$, $\Lambda=3.0 \mathrm{~m} \mathrm{sec}-1 / 100 \mathrm{mb}$ and $\Lambda^{\prime}=0.25 \mathrm{~m}$ $\mathrm{sec}^{-1} /(100 \mathrm{mb})^{2}$.

from Fig. 3, 4-level model and 8-level model make the disturbance of $L=2000 \mathrm{~km}$ unstable in a baroclinic atmosphere with $\Lambda=3.0 \mathrm{~m}$ $\mathrm{sec}^{-1} / 100 \mathrm{mb}$ if the effect of $\Lambda^{\prime}$ is not taken into account. Therefore a stabilizing effect can be inferred. Although the experiment has been made only for limited cases, the following conclusion seems to be derived concerning the effects of higher modes in the vertical profile of general flow: a convex type distribution has destabilizing effect and a concave type distribution has stabilizing effect. Considering the thermal wind relationship, it can also be stated that a disturbance becomes more unstable if the static stability increases towards north and vice versa. The author has ever dealt the matter with a simplified model and obtained a qualitatively similar conclusion (Matsumoto, 1960).

\section{Level model and parameter model}

There are several approaches in representing the baroclinic structure of the atmosphere. Generally accepted idea of parameter models is to find out proper parameters which minimize the error of governing equations. The most reasonable and consistent way of constructing such parameters would undoubtedly be to expand the meteorological elements by some kind of orthogonal functions. However, the number of freedom is practically limited to a very small number and it is rather questionable whether we can obtain a set of parameters which have physically meaningful context. So far as we stay within the quasigeostrophic approximation, there could be some other conventional methods in which vertical derivatives are estimated as correctly as possible with the aid of experimental knowledge concerning the structure of baroclinic disturbances.

Let us take the matter hypothetically in the following way. Suppose that our observations are sufficiently accurate on a certain number of meteorologically significant levels and the procedure in the atmosphere is exactly governed by quasi-geostrophic vorticity equation and $\omega$-equation such as given for linearized case by

$$
\begin{aligned}
& \frac{\partial \nabla^{2} z}{\partial t}+U \frac{\partial V^{2} z}{\partial x}-\frac{f^{2}}{g} \omega_{p}=0 \\
& \frac{f^{2}}{g} \omega_{p p}+\sigma \nabla^{2} \omega=2 U_{p} \frac{\partial V^{2} z}{\partial x}
\end{aligned}
$$

Then our problem is how to estimate the divergence effect as correctly as possible in order to obtain an accurate forecast. The divergence which cannot be observed directly is related through (17) to the vorticity field which can be measured on predetermined $n$ information levels. If we can find an adequate interpolation form for the vertical distribution of the vorticity field, we can obtain a continuous $\omega$ field as a solution of differential equation (17), from which the divergence can be estimated. Thus our conventional technique is to be regarded simply as interpolation method. As to the method obtaining a solution of (17), we can also apply the parametric representation for $\omega$ distribution using a similar interpolation formula which may or may not be different from that for the vorticity field.

The practical procedure of integration will be given in the following. Let us define

$$
z=\sum_{i=0}^{n-1} A_{i}(p) z_{i}
$$

where $z_{i}$ 's are parameters defined by $z$ field on $n$ information levels and $A_{i}(p)$ 's are polynomials of pressure $p$ with $i$ degree. We regard this expression simply as an interpolation formula. Physical significance of parameters $z_{i}$ 's will be given later in association with the choice of polynomials $A(p)$ 's. Similarly let us define

$$
W=\sum_{i=0}^{m-1} B_{i}(p) W_{i}
$$

where $W=f^{2} \omega / g$ and the number of parameters 
$W_{i}$ 's is $m$. The polynomials $B_{i}(p)$ 's and $m$ are not necessarily the same as $A_{i}(p)$ 's and $n$. Taking into account the boundary conditions at the top and the bottom of air column under consideration, the number of independent parameters for $W$ field is $m-2$. Therefore (19.1) is rewritten

$$
W=\sum_{i=2}^{m-1} C_{i}(p) W_{i}
$$

where $m-2$ parameters with higher modes are retained and $C_{i}(p)$ 's are polynomials of degree $i$ which are generated from $B_{i}(p)$ 's.

We obtain from (19.2)

$$
\begin{gathered}
W_{p}=\sum_{i=2}^{m-1} C_{i}^{\prime}(p) W_{i} \\
W_{p p}=\sum_{i=2}^{m-1} C_{i}^{\prime \prime}(p) W_{i}
\end{gathered}
$$

where primes denote the differentiation with respect to $p$. Substituting (19:2) and (21) into (17), we have

$$
\sum_{i=2}^{m-1} C_{i}^{\prime \prime}(p) W_{i}+S(p) \sum_{i=2}^{m-1} C_{i}(p) \nabla^{2} W_{i}=F
$$

where $S(p)=g \sigma / f^{2}$. Obviously (22) does not hold throughout the atmosphere. However our method of approximation is to find out higher modes in $W$ distribution so as to estimate the effect of divergence correctly as possible. $m-2$ parameters $W_{2}, W_{3} \cdots \cdots, W_{m-1}$ are to be solved by assuming that (22) holds at least on $m-2$ levels. Thus we have the following simultaneous differential equation written in a matrix form

$$
\mathfrak{A} \nabla^{2} \xi+\mathfrak{B} \xi=\eta
$$

Matrix $\mathfrak{A}, \mathfrak{B}$ and vectors $\xi, \eta$ are given by

$$
\begin{aligned}
\mathfrak{A}_{i j} & =S\left(p_{j}\right) C_{i}\left(p_{j}\right) \\
\mathfrak{B}_{i j} & =C_{i}^{\prime \prime}\left(p_{j}\right) \\
\xi_{i} & =W_{i} \\
\eta_{j} & =F\left(p_{j}\right)
\end{aligned}
$$

where $p_{j}$ is the pressure coordinate at which (17) is assumed to hold. Eq. (23) is solved in a similar manner as is applied by Charney et. al. (1953). Denoting the eigen value of the matrix $\mathfrak{A}^{-1 \mathfrak{B}}$ and the associated eigen vector by $\lambda^{i}$ and $\zeta^{i}$ respectively, (23) is rewritten

$$
\nabla^{2} \zeta^{i} \xi+\lambda^{i} \zeta^{i} \xi=\xi^{i}\left(\mathfrak{A}^{-1} \eta\right) \quad(i=1,2, \cdots \cdots, m-2)
$$

As is well known, (24) is the Helmholtz

type differential equation and can be solved with respect to the vector product $\zeta \xi \xi$ provided that we can obtain positive value for $\lambda^{i}$ 's. Letting $h_{i}$ denote the solution of $m$-2 differential equations (24), we have $m-2$ simultaneous equations

$$
\zeta^{i} \xi=h_{i}
$$

from which parameters $W_{2}, W_{3}, \cdots \cdots, W_{m-2}$ are determined.

Eqs. (20) and (21) may be regarded as a kind of generalized differencing formula. Integration is made by marching process. Now let us compare the new designated parameter model and the ordinary level model using 4-parameter and 4-level linearized quasi-geostrophic models.

Specifying four information levels by $p_{1}, p_{2}, p_{3}$ and $p_{4}$ as is shown in Fig. 8 , the vorticity field is represented by

$$
z=A_{0}(p) z_{0}+A_{1}(p) z_{1}+A_{2}(p) z_{2}+A_{3}(p) z_{3}
$$

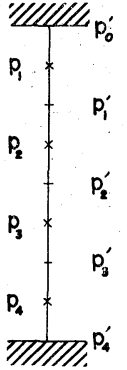

Fig. 8.

where $A_{i}(p)$ 's are constructed successively as follows :

$$
\begin{aligned}
& A_{0}(p)=1 \\
& A_{1}(p)=\frac{p-p_{1}}{p_{3}-p_{1}} \\
& A_{2}(p)=\frac{\left(p-p_{1}\right)\left(p-p_{3}\right)}{\left(p_{2}-p_{1}\right)\left(p_{2}-p_{3}\right)} \\
& A_{3}(p)=\frac{\left(p-p_{1}\right)\left(p-p_{2}\right)\left(p-p_{3}\right)}{\left(p_{4}-p_{1}\right)\left(p_{4}-p_{2}\right)\left(p_{4}-p_{3}\right)}
\end{aligned}
$$

Similar expressions are already applied in the author's previous paper (Matsumoto, 1960). Then the relation between the parameters $z_{i}$ 's and the observed field at each of information levels is given by

$$
\begin{aligned}
& z\left(p_{1}\right)=z_{0} \\
& z\left(p_{2}\right)=z_{0}+\frac{1}{2} z_{1}+z_{2} \\
& z\left(p_{3}\right)=z_{0}+z_{1} \\
& z\left(p_{4}\right)=z_{0}+\frac{3}{2} z_{1}-3 z_{2}+z_{3}
\end{aligned}
$$

or

$$
\begin{aligned}
& z_{0}=z\left(p_{1}\right) \\
& z_{1}=z\left(p_{3}\right)-z\left(p_{1}\right) \\
& z_{2}=z\left(p_{2}\right)-\frac{1}{2}\left[z\left(p_{1}\right)+z\left(p_{3}\right)\right] \\
& z_{3}=z\left(p_{4}\right)-3 z\left(p_{3}\right)+3 z\left(p_{2}\right)-z\left(p_{1}\right)
\end{aligned}
$$


for the equi-pressure-depth spacing of information levels.

The physical meaning of parameters is now clear, i. e., $z_{0}$ corresponds to the vorticity field on the equivalent barotropic level, $z_{1}$ is related to the thickness field, $z_{2}$ to the static stability field and $z_{3}$ to static stability difference between layers. The vertical velocity field is also expanded into a similar series in terms of $p_{0}^{\prime}, p_{1}^{\prime}, \cdots \cdots, p_{4}^{\prime}$ shown in Fig. 8 at which the $\omega$-equation (17) is assumed to hold. This formulation is preferred so as to keep the similarity between two models as far as possible. Keeping in mind the boundary condition $W\left(p_{0}{ }^{\prime}\right)=W\left(p_{4}{ }^{\prime}\right)=0$, we have three parameters in representing $W$ field.

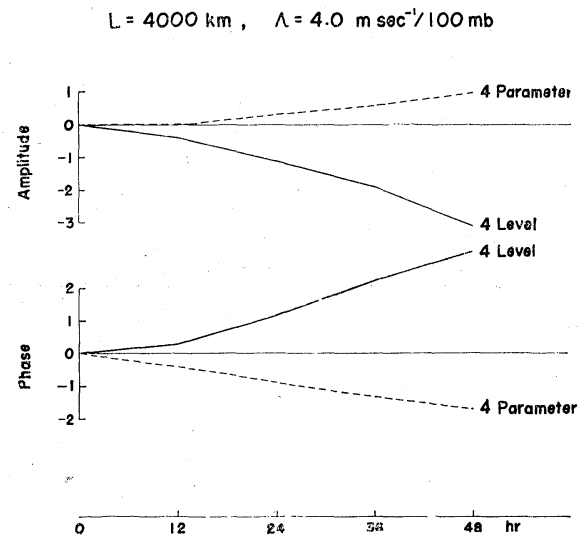

Fig. 9. Time change of error in amplitude and phase angle of 4-parameter model (broken line) and 4-level model (full line) for the unstable disturbance $(L=4000$ $\mathrm{km}, \Lambda=4.0 \mathrm{~m} \mathrm{sec}-1 / 100 \mathrm{mb})$.

Integrations are made for both of 4-parameter and 4-level model basing on the same initial condition and boundary condition, and the results are compared with those obtained by means of 8-level model which are considered to give more acurate solutions. Fig. 9 gives an example by which a disturbance having the wave length of $4000 \mathrm{~km}$ is considered in the atmosphere where the vertical wind shear is $4.0 \mathrm{~m} \mathrm{sec}^{-1} / 100 \mathrm{mb}$. This figure shows how the amplitude and the phase angle deviate from the presumed true values. It is seen that 4-parameter model gives better approximation to 8-level than 4-level model for both of amplitude and phase angle, in spite of the fact that the mathematical consistency is not yet verified on the newly proposed parameter model.

\section{Summary and conclusions}

The baroclinic behavior of multi-level models was studied by means of linearized quasigeostrophic models. Principal factors to be considered would be a) the numbers of levels, b) the vertical distribution of the static stability and c) the vertical profile of the general flow. If we divide the atmosphere into more layers, the vertical truncation errors will be diminished. The instability diagram does change among models with different number of levels, but the change does not seem to bear any practically significant improvement simply by increasing the number of levels. More important contributions originate from the fact that the less stable stratification in the lower part of the atmosphere thus comes to be taken into account. A low static stability makes the smaller scale disturbances to predominate. A series of numerical integrations were performed experimentally, and it was found that the interaction between layers is not very strong so that the predominating scale of disturbance in the upper atmosphere and the lower atmosphere is characterized almost independently by the local static stability if the number of levels applied is sufficiently large.

It is well known that the disturbance in the unstable regime tends to have a certain tilt of trough or ridge and a phase velocity regardless of its initial configuration. The'stable disturbance in a baroclinic atmosphere, on the other hand, is likely to be shifted with the local velocity of general flow, resulting in a growth of phase difference with time between layers according to the vertical wind shear. It turns out to bear a periodical change of amplitude, if we deal with the atmosphere by means of level models, because the phase difference between adjacent layers comes to be equal to the wave length in due course of time and thus return to the initial configuration again. Thus numerical integrations show that this appearant period of stable oscillation is longer for larger number of layers in our multiple-level model because the difference in wind velocity between layers becomes smaller under a given wind shear. 
The effect of the second derivative in the vertical profile of general flow was also studied. Although integrations were made only for limited cases, it is concluded that a destabilizing effect of convex type and a stabilizing effect of concave type distribution can be inferred. It may be said that the larger wind shear in the lower atmosphere has an influencing effect, whereby the lower static stability is also favorable to provide destabilizing effect.

A multiple parameter model is proposed basing on a rather simple idea of interpolation technique to estimate the divergence effect as precisely as possible. Although the mathematical consistency is not yet verified, some numerical computations seem to show its usefulness at least in linearized models.

\section{Acknowledgement}

The author wishes to thank Dr. H. Arakawa for his encouragments and Miss S. Hiraoka for her assistance in numerical computations and drafting the figures.

\section{Appendix}

Eq. (13) together with (14) can be written down in the following manner:

$$
\begin{gathered}
-\alpha_{1} W_{1}+W_{2}=F_{1} \\
W_{1}-\alpha_{2} W_{2}+W_{3}=F_{2} . \\
\cdots \cdots \cdots \cdots \\
W_{K-2}-\alpha_{K-1} W_{K-1}=F_{K-1}
\end{gathered}
$$

Assuming that $W_{1}$ is given by $\xi$, we can express $W_{k}$ 's in term of $\xi$, i.e.

$$
\begin{array}{ll}
W_{1}=\xi & =G_{0}+\beta_{0} \xi \\
W_{2}=F_{1}+\alpha_{1} W_{1} & =G_{1}+\beta_{1} \xi \\
W_{3}=F_{2}+\alpha_{2} W_{2}-W_{1} & =G_{2}+\beta_{2} \xi
\end{array}
$$

$$
\begin{aligned}
W_{k}=F_{k-1}+\alpha_{k-1} W_{k-1}-W_{k-2} & =G_{k-1}+\beta_{k-1} \xi \\
\ldots \ldots \ldots \ldots \ldots . . & \\
W_{K}=F_{K-1}+\alpha_{K-1} W_{K-1}-W_{K-2} & =G_{K-1}+\beta_{K-1} \xi \\
& =0
\end{aligned}
$$

where $G_{k}$ and $\beta_{k}$ are obtained successively by the following recurence formulae:

$$
\begin{aligned}
G_{k} & =F_{k}+\alpha_{k} G_{k-1}-G_{k-2} \\
\beta_{k} & =\alpha_{k} \beta_{k-1}-\beta_{k-2}
\end{aligned}
$$

with $\beta_{0}=1, \beta_{1}=\alpha_{1}$ and $G_{0}=0, G_{1}=F_{1}$.

Thus having $\xi=-G_{k-1} / \beta_{k-1}$ for the given $\alpha_{k}$ 's and $F_{k}$ 's the solution $W_{k}$ 's are easily constructed.

\section{References}

Ogura, Y., 1957: Wave solutions of the vorticity equation for the $2 \frac{1}{2}$-dimensional model. J. Meteor., 14, 60-64.

Matsumoto, S., 1960: A role of static stability as a parameter of atmospheric models. Journ. Met. Soc. Japan, 38, 239-249.

Matsumoto, S., 1962: A numerical experiment using five-level geostrophic model. (to be published in Proceedings of the International Symposium on Numerical Weather Prediction in Tokyo).

Charney, J.G. and N.A. Phillips, 1953: Numerical integration of the quasi-geostrophic equations for barotropic and simple baroclinic flows. J. Meteor., 10, 71-99.

Saito, N., 1962: The results of numerical forecasting with the 4-level baroclinic model. (to be published in Proceedings of the International Symposium on Numerical Weather Prediction in Tokyo).

Eliassen, A., 1953: Simplified dynamic models of the atmosphere, designed for the purpose of numerical weather prediction. Tellus, 4, 145 $-156$.

Charney, J.G., 1962: Integration of the primitive and balance equations. (to be published in Proceedings of the International Symposium on Numerical Weather Prediction in Tokyo). 


\title{
多層地衡風 モデルの傾圧不安定性について
}

\author{
松本，誠一 \\ (気 象 研 究 所).
}

地衡風モデルの適用性を見るための一つの手がかりとして，層の数をふやしたときと傾圧不安定性がぞう変わるか を調べた。不安定波の波長和よび增巾率は鉛直安定度の関数であるが，層の数を゙多くとつた場合，上下の層の異なつ た鉛直安定度からろける影響は比較的小さく，第一義的にはその高度に招ける安定度によつて傾圧不安定波が支配さ れる。したがつて層の数を多くとることは，低層に招ける安定性の弱い大気中に括ける性格をよりょく記述するとい ろ点汇重要性がある。

基本場の風が鉛直飞曲率をもつている場合の影響を $2 ， 3$ の例飞ついて数値的に調べた。

補間方式という考えを基礎としたパラメーターモデルを考案し，4 パラ゙メーターモデルとこれに対応する 4 層モデ ルとを比較した。

\section{Corrigenda}

\begin{tabular}{c|c|c|c|c|c}
\hline \hline & page & column & line & for & read \\
\cline { 1 - 3 } & 351 & right & 3 from bottom & $L=13.3 \frac{A_{u}}{A_{T}}$ & $\begin{array}{c}L=13.3 \frac{A_{u}{ }^{2}}{A_{T}} \\
(\tau / \rho)\end{array}$ \\
& & Fig. 5 & $(\tau / \rho)^{\frac{1}{2}}$ \\
\hline
\end{tabular}

